\title{
Metastatic chorioretinal abscess: an unusual complication of pyogenic liver abscess
}

\author{
(1) Gokul Krishnan, (1) Sharath Madhyastha P., (1) Raviraja V. Acharya, (1) Sulatha Bhandary, (1) Charan Thej \\ Reddy
}

Manipal Academy of Higher Education, Kasturba Medical College, Department of Medicine, Manipal, India

\section{Date submitted: \\ 05.07.2020}

Date accepted:

23.11.2020

Online publication date:

15.09.2021

\section{Corresponding Author:}

Sharath Madhyastha P. M.D., Assoc.

Prof., Manipal Academy of Higher

Education, Kasturba Medical College,

Department of Medicine, Manipal,

India

dr.sharathymc@gmail.com

ORCID:

orcid.org/0000-0003-2821-3473

Keywords: Liver abscess, chorioretinal abscess, diabetes mellitus

\begin{abstract}
Liver abscesses are the most common type of visceral abscess, which accounts for around one half of all visceral abscesses. Rarely, the liver abscess can metastasize to distant organs and diabetes mellitus is an important risk factor for septic metastasis. We here present one such rare case of chorioretinal abscess in a diabetic male with pyogenic liver abscess. A 54-year-old diabetic male presented with the complaints of fever, pain abdomen and blurring lasting for one week. After detailed evaluation, he was diagnosed to have pyogenic liver abscess with chorioretinal abscess. He was treated with parenteral and topical antibiotic for eyes, strict glycemic control and ultrasound guided aspiration of liver abscess. The patient symptomatically improved and improvement in vision was also noted.
\end{abstract}

\section{Introduction}

Liver abscesses are the most common type of visceral abscess, which accounts for around one half of all visceral abscesses. In Asia, the enteric Gram-negative bacilli, particularly Escherichia coli and Klebsiella pneumoniae, are the most common organisms. Rarely, the liver abscess can metastasize to distant organs. Diabetes is an important risk factor for septic metastasis due to increased chances of bacteremia in them. Metastatic chorioretinal abscess is a rare subset of metastatic endophthalmitis.

\section{Case Presentation}

A54-year-old diabetic man came to the outpatient department with seven-day history of fever, cough and abdominal pain. Fever was high grade, intermittent with chills and rigors. Cough was mild and non-productive. His abdominal pain was severe, was located in the right upper quadrant and increased with change of posture. He was a non-smoker and occasionally consumed alcohol. The examination revealed mild icterus and right hypochondriac tenderness. Other systemic examinations were normal. His initial blood investigations revealed leukocytosis $(16,300 / \mu \mathrm{L})$ and poorly controlled diabetes with glycated hemoglobin of $12 \%$. There was conjugate hyper bilirubinemia with total bilirubin of $3.2 \mathrm{mg} / \mathrm{dL}$ (normal: $0.2-1.1 \mathrm{mg} / \mathrm{dL}$ ) and direct bilirubin of $2.3 \mathrm{mg} / \mathrm{dL}$ (normal: $0.0-0.2 \mathrm{mg} / \mathrm{dL}$ ). Liver enzymes were also elevated; alanine aminotransferase of $181 \mathrm{U} / \mathrm{L}$ (normal level: $12-38 \mathrm{U} / \mathrm{L}$ ), aspartate aminotransferase of $187 \mathrm{U} / \mathrm{L}$ (normal level: $15-37 \mathrm{U} / \mathrm{L}$ ), alkaline phosphatase of $110 \mathrm{U} / \mathrm{L}$ (normal level: 45-115U/L. Renal function tests and serum electrolytes were normal. Chest X-ray (Figure 1) showed an elevated right 


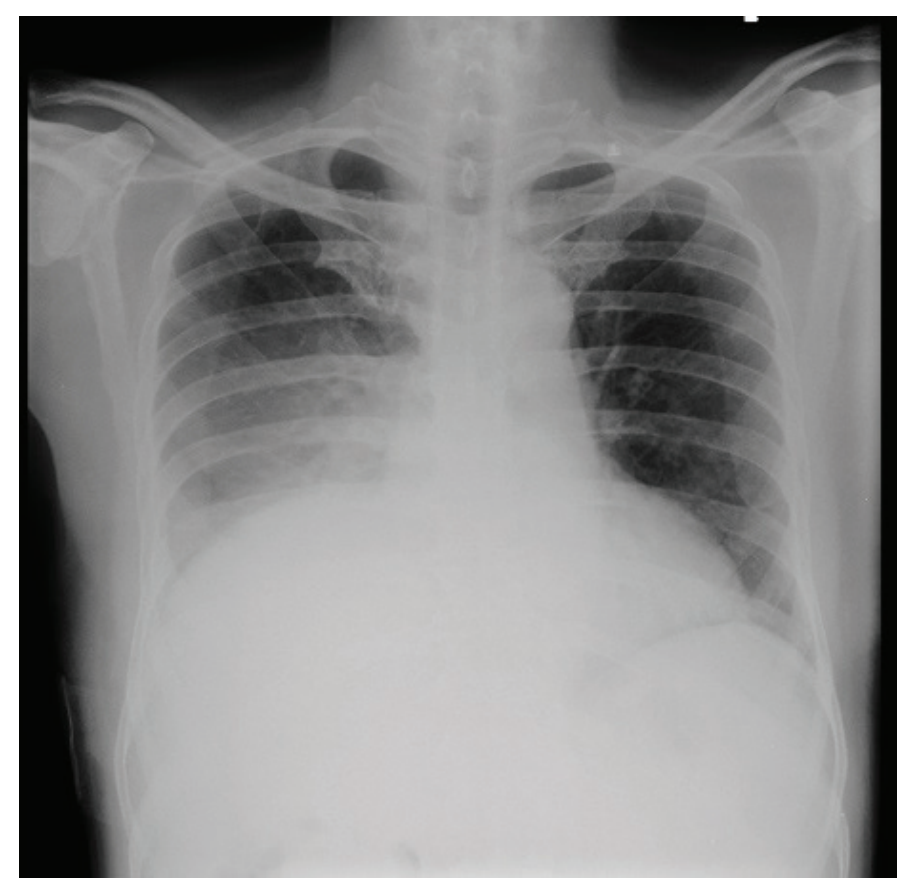

Figure 1. Frontal chest radiograph showing the elevation of right hemidiaphragm

hemi-diaphragm with mild right sided pleural effusion. Contrast enhanced computed tomography (CECT) of the abdomen (Figure 2) revealed hypo-dense lesion with irregular walls in the liver (measuring $7.1 \times 6.5 \times 4.6 \mathrm{~cm}$ ), involving segment VII and VIII with multiple air pockets within, suggestive of abscess.

The abscess was drained with a pig tail catheter, which was inserted under ultrasound guidance. Pus was sent for culture which grew multi drug resistant Escherichia coli. Even the blood culture grew the same organism sensitive only to amikacin and meropenem.

On day four of admission, the patient started noticing blurring of vision in his left eye. Ophthalmology evaluation showed decreased visual acuity (counting fingers at 3 meters) in the left eye. Vitreous exudates and hemorrhage could be seen on fundus examination. A yellowish circular deep choroidal lesion with retinal oedema and sheathing of the retinal blood vessels,

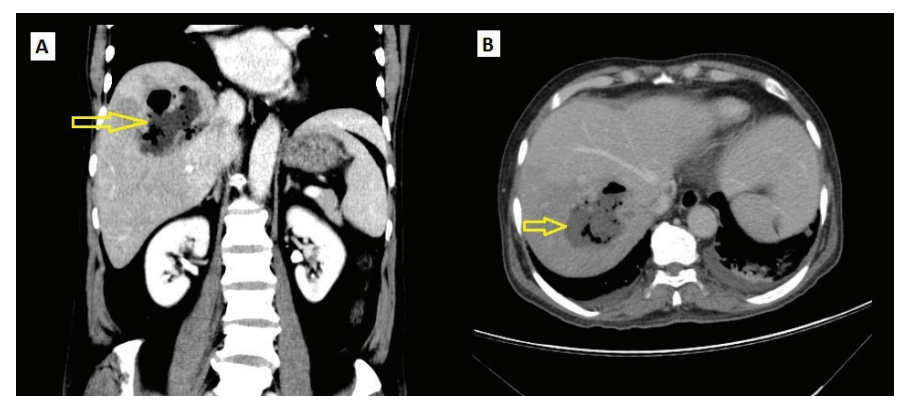

Figure 2. Contrast enhanced computed tomography abdomen (A: coronal, B: axial views) showing hypo-dense lesion with irregular walls in the liver (measuring $7.1 \times 6.5 \times 4.6 \mathrm{~cm}$ ) involving segment VII and VIII with multiple air pockets within, suggestive of abscess which was noted as suggestive of chorioretinal abscess, were observed (Figure 3).

The patient received intravenous (amikacin and meropenem) and topical (tobramycin) antibiotics for two weeks, with which he improved symptomatically.

Liver abscesses are the most common type of visceral abscess, which account for around one half of all visceral abscesses. In Asia, the enteric Gram-negative bacilli, particularly Escherichia coli and Klebsiella pneumoniae, are the most common organisms. Whereas in the United States, Streptococci (in particular, the Streptococcus milleri group) were the most common pathogen of pyogenic liver abscess. Most common clinical features are fever and abdominal pain with or without other constitutional symptoms (1).

Rarely, the liver abscess can metastasize to distant organs. Diabetes is an important risk factor for septic metastasis due to increased chances of bacteremia in them. Metastatic chorioretinal abscess is a rare subset of metastatic endophthalmitis. Endophthalmitis refers to a condition characterized by inflammation of inner structures of the eyeball, which includes anterior as well as posterior segment structures. Infectious cause may be endogenous or exogenous. Endogenous/ metastatic endophthalmitis results from bacteremia secondary to an infective foci in body (2).

Clinical features include ocular pain, redness, lacrimation and photophobia. Panophthalmitis and vision loss are among

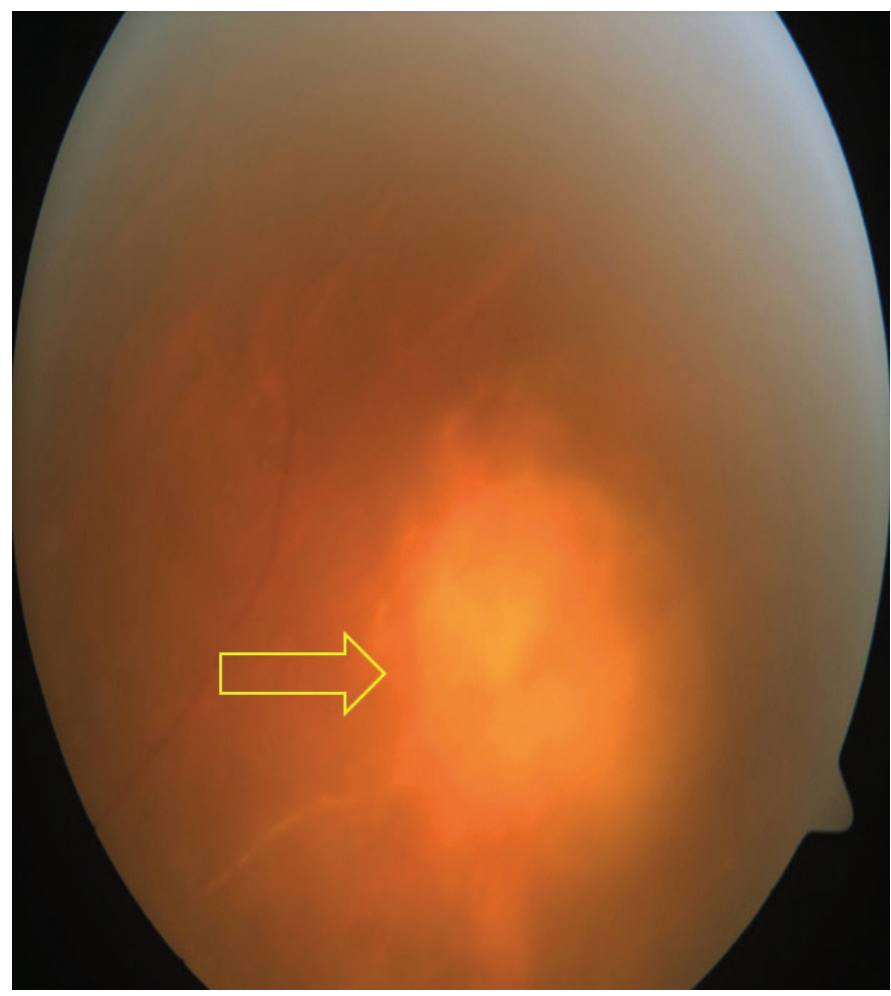

Figure 3. Fundus picture showing focus of retinitis temporally with features suggestive of chorioretinal abscess 
the dreaded complications of this condition. Antibiotics are the principle mode of treatment. Usually topical antibiotics are initiated first, followed by intravitreal antibiotics. Supportive therapy like cycloplegics for pain relief and intraocular pressure lowering drugs are also used (3).

If it complicates to panophthalmitis, then there is the risk of orbital cellulitis, cavernous sinus thrombosis and meningitis. Immediate evisceration has to be performed under the cover of broad spectrum antibiotics to avoid further spread. Early eye evaluation and continued intravenous antibiotics are important treatment aspects. Any delay in intervention may result in permanent loss of vision $(4,5)$.

Our patient had poorly controlled diabetes as a risk factor and presented with classical symptoms of liver abscess. Here the organism was Escherichia coli and the patient received both systemic and topical antibiotics. Intravitreal antibiotic therapy was not given as there was dramatic improvement in visual acuity within three days of the initiation of the treatment. Followup images taken after three weeks showed significant resolution of both liver abscess and chorioretinal abscess (Figure 4).

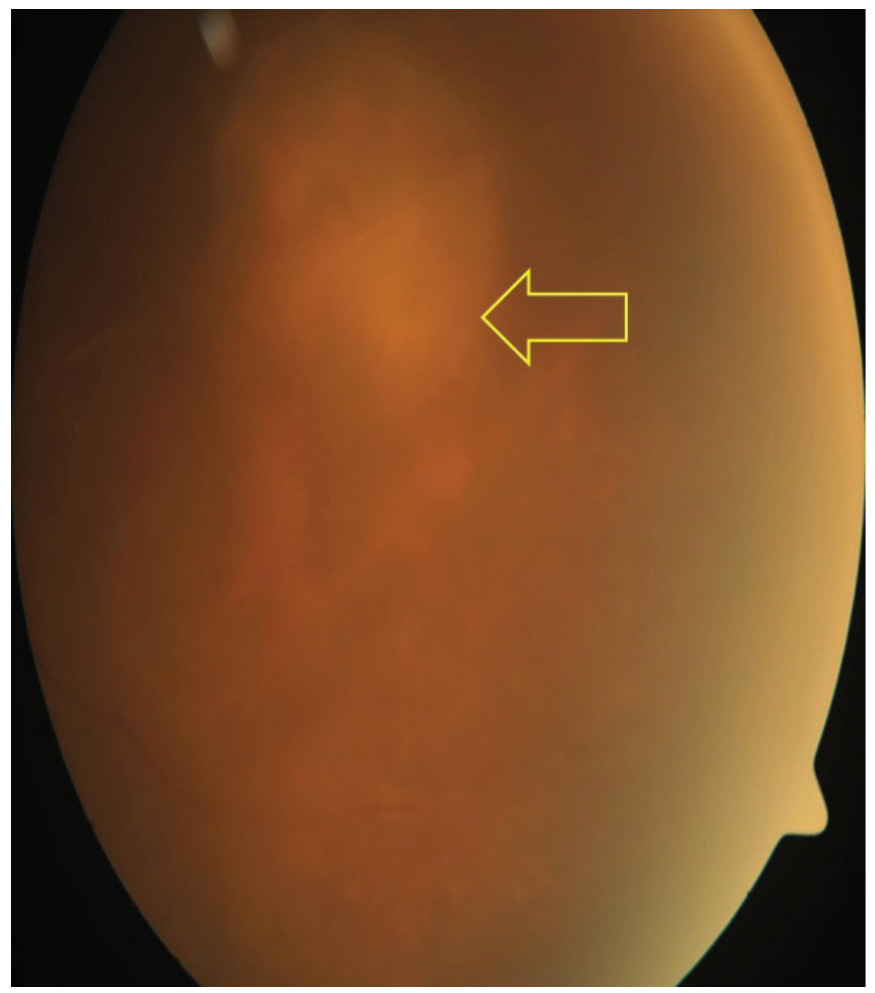

Figure 4. Fundus picture showing a more localized and resolving lesion

\section{Conclusion}

Metastatic chorioretinal abscess is an unusual complication of pyogenic liver abscess. Poorly controlled diabetes is an important risk factor. Prompt diagnosis and early systemic antibiotic therapy are cornerstone to prevent its progression to fulminant endophthalmitis and subsequent permanent blindness.

\section{Acknowledgments}

We acknowledge the department of radiology and ophthalmology for providing CECT abdomen and fundus images respectively.

\section{Ethics}

Informed Consent: Consent form was filled out by all participants.

Peer-review: Internally peer-reviewed.

\section{Authorship Contributions}

Surgical and Medical Practices: R.V.A., S.B., Concept: S.M.P., R.V.A., Design: S.M.P., Data Collection or Processing: S.B., C.T.R., Analysis or Interpretation: S.M.P., Literature Search: G.K., C.T.R., Writing: G.K.

Conflict of Interest: No conflict of interest was declared by the authors.

Financial Disclosure: The authors declared that this study received no financial support.

\section{References}

1. Meddings L, Myers RP, Hubbard J, et al. A populationbased study of pyogenic liver abscesses in the United States: incidence, mortality, and temporal trends. Am J Gastroenterol. 2010;105:117-124.

2. Tsai TH, Peng KL. Metastatic endophthalmitis combined with subretinal abscess in a patient with diabetes mellitus-a case report. BMC Ophthalmol. 2015;15:105.

3. Jackson TL, Eykyn SJ, Graham EM, Stanford MR. Endogenous bacterial endophthalmitis: a 17-year prospective series and review of 267 reported cases. Surv Ophthalmol. 2003;48:403-423.

4. Han SH. Review of hepatic abscess from Klebsiella pneumoniae. An association with diabetes mellitus and septic endophthalmitis. West J Med. 1995;162:220-224.

5. Liu YC, Cheng DL, Lin CL. Klebsiella pneumoniae liver abscess associated with septic endophthalmitis. Arch Intern Med. 1986;146:1913-1916. 